\title{
The pharmacokinetic challenge of treating invasive aspergillosis complicating severe influenzae assisted by extracorporeal membrane oxygenation
}

\author{
Hadrien Winiszewski ${ }^{1,6^{*}}$ (D) Anne-Claire Rougny², Jennifer Lagoutte-Renosi ${ }^{3}$, Laurence Millon ${ }^{4,6}$, Gilles Capellier ${ }^{1,5,6}$, \\ Jean-Christophe Navellou', Gael Piton ${ }^{1,6}$ and Anne-Laure Clairet ${ }^{2}$
}

Invasive aspergillosis (IA) complicates 19\% of patients requiring ICU admission for severe influenza [1]. Despite recent development of isavuconazole, voriconazole remains the first therapeutic option for IA among patients without hematological malignancy [2]. However, among patients requiring the use of extracorporeal membrane oxygenation (ECMO) as rescue therapy for extremely severe acute respiratory failure [1], the use of ECMO per se has been reported as a cause of sequestration of voriconazole on the membrane. This phenomenon could induce low plasma concentrations of voriconazole and, therefore, limited efficacy [3].

A 57-year-old man with no significant medical history was admitted to our ICU for severe influenza. After intubation, broncho-alveolar lavage revealed the presence of Aspergillus fumigatus. Galactomannan antigen and PCR for Aspergillus DNA were also positive in epithelial lining fluid. A computed tomography scan was consistent with the diagnosis of IA and intravenous voriconazole was started with usual doses. After one week, despite maximal respiratory management, veno-venous ECMO (CardioHelp MAQUET system, Getinge society) was introduced because of refractory respiratory acidosis. Therapeutic drug monitoring was performed targeting a residual concentration between 2 and $6 \mathrm{mg} / \mathrm{L}$. Weaning of ECMO was not possible and the patient stayed under ECMO support for 4 months. As a consequence, a total of five ECMO membranes were needed. We observed after every membrane change

\footnotetext{
* Correspondence: hwiniszewski@chu-besancon.fr

${ }^{1}$ Medical Intensive Care Unit, Besançon, France

${ }^{6}$ Research Unit EA 3920 and SFR FED 4234, University of Franche Comté,

Besançon, France

Full list of author information is available at the end of the article
}

a drop of voriconazole plasma concentration, with the need to increase the dose (Fig. 1). Because of the high pharmacokinetic variability, a combination therapy of voriconazole with liposomal amphotericin B was started from week 2. Despite such combination therapy and therapeutic drug monitoring, failure to wean the patient from ECMO led to care withdrawal after 5 months.

In the present case, the diagnosis of refractory IA was considered since lung infiltrates and endo-tracheal aspirate cultures of Aspergillus fumigatus persisted during the entire ICU stay. In addition, galactomannan antigen was still detected in epithelial lining fluids after 18 weeks of treatment by voriconazole. Such treatment failure was probably linked to frequent voriconazole underdosing. Indeed, 17 out of the 32 times therapeutic drug was monitored showed voriconazole concentrations under the target of $2 \mathrm{mg} /$ $\mathrm{L}$ despite high dose administration (until $12 \mathrm{mg} / \mathrm{kg}$ twice a day).

Sequestration of voriconazole, a lipophilic drug, on the ECMO membrane is a well-known phenomenon [3]. As no drug-drug interaction was detected in the present case, sequestration of voriconazole was likely the main reason leading to such pharmacokinetic variability. After 2 or 3 weeks of membrane use, the membrane was well saturated by voriconazole, resulting in sufficient and stable plasma concentrations in the patient. However, immediately after the membrane change, intense adsorption of voriconazole on the new membrane was likely responsible for the drop in plasma concentration. Several days of high voriconazole dosing were then required before the plasma concentration reached the target concentration. 


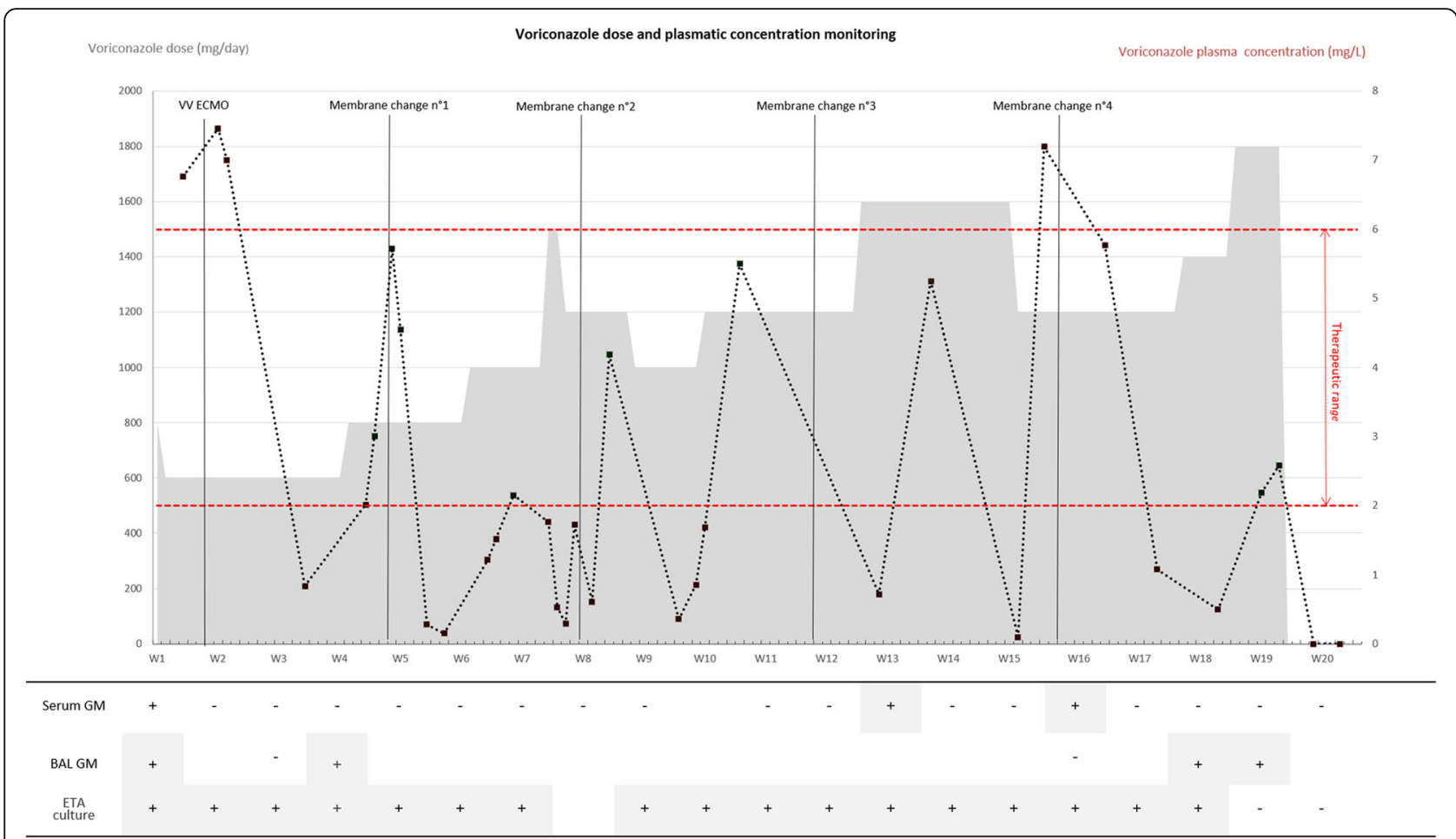

Fig. 1 Evolution of voriconazole dose and plasma residual concentration during the ICU stay. The $x$-axis corresponds to time (weeks). The left $y$-axis corresponds to the voriconazole dose (mg/day). The gray shaded area corresponds to the dose evolution of voriconazole. The right $y$-axis corresponds to the voriconazole plasma concentration (mg/L). Black dotted line corresponds to evolution of voriconazole plasma concentration. Upper and lower red dotted line correspond to the therapeutic range of voriconazole plasma concentration (i.e., 2-6 mg/L). The lower part of the figure corresponds to evolution of serum galactomannan antigen (serum GM), broncho-alveolar lavage galactomannan antigen (BAL GM), and endo-tracheal aspirates culture for aspergillus (ETA culture)

Given the high number of IA complicating influenza, and the increasing development of the ECMO technique for refractory acute respiratory failure, the described situation might become frequent. Data regarding the pharmacokinetics and efficacy of other antifungal drugs, such as isavuconazole, during ECMO therapy are urgently needed.

\section{Abbreviations}

CT: Computed tomography; ECMO: Extracorporeal membrane oxygenation; IA: Invasive aspergillosis; ICU: Intensive care unit; PCR: Polymerase chain reaction

\section{Acknowledgments}

Not applicable.

\section{Funding}

Not applicable.

\section{Availability of data and materials}

The datasets are available from the corresponding author on reasonable request.

\section{Authors' contributions}

$H W, A-C R$, and $A-L C$ wrote the manuscript and performed the literature search. JL-R performed therapeutic drug monitoring. LM performed galactomannan antigen assay. GC, J-CN, and GP analyzed the data and consistently improved the manuscript. All authors accepted the final version of the manuscript.
Ethics approval and consent to participate

Not applicable.

\section{Consent for publication}

This was a retrospective observation of an individual patient. According to the French legislation, neither informed consent nor approval of the ethics committee were required. However, general information of the families was made in the ICU by a poster explaining that data concerning their next of kin might be used later for scientific analyses, and could be published with anonymized data unless they express their opposition. The patient died in the ICU. No opposition was expressed by the family of the patient.

\section{Competing interests}

The authors declare that they have no competing interests.

\section{Publisher's Note}

Springer Nature remains neutral with regard to jurisdictional claims in published maps and institutional affiliations.

\section{Author details}

${ }^{1}$ Medical Intensive Care Unit, Besançon, France. ${ }^{2}$ Department of Pharmacy, Besançon, France. ${ }^{3}$ Department of Clinical Pharmacology, Besançon, France. ${ }^{4}$ Department of Clinical Mycology, Besançon, France. ${ }^{5}$ Department of Epidemiology and Preventive Medicine, School of Public Health and Preventive Medicine, Faculty of Medicine, Nursing and Health Sciences, Clayton, Australia. ${ }^{6}$ Research Unit EA 3920 and SFR FED 4234, University of Franche Comté, Besançon, France. 
Received: 23 October 2018 Accepted: 28 November 2018

Published online: 22 December 2018

\section{References}

1. Schauwvlieghe AFAD, Rijnders BJA, Philips N, et al. Invasive aspergillosis in patients admitted to the intensive care unit with severe influenza: a retrospective cohort study. Lancet Respir Med. 2018;6(10):782-92.

2. Ullmann AJ, Aguado JM, Arikan-Akdagli S, et al. Diagnosis and management of Aspergillus diseases: executive summary of the 2017 ESCMID-ECMM-ERS guideline. Clin Microbiol Infect Off Publ Eur Soc Clin Microbiol Infect Dis. 2018;24(Suppl 1):e1-e38.

3. Mehta NM, Halwick DR, Dodson BL, et al. Potential drug sequestration during extracorporeal membrane oxygenation: results from an ex vivo experiment. Intensive Care Med. 2007;33:1018-24. 